\title{
Performance Degradation Due To Polarization Mode Dispersion in a Coherent Optical System with Pilot Carrier
}

\author{
Ming-Seng Kao and Jingshown Wu
}

\begin{abstract}
The use of pilot carrier in a coherent optical communication system can simplify the receiver; however, the polarization mode dispersion caused by fiber birefringence may degrade the system performance. Here we use the formulation of principal states of polarization and provide a simple expression to evaluate the performance degradation.
\end{abstract}

\section{INTRODUCTION}

ATHOUGH coherent optical communications offer signifiAcant sensitivity improvement over intensity modulated systems, the need of a local laser oscillator and the inherent polarization problem inevitably result in a complex and costly receiver. An elegant approach to simplify the receiver is to transmit a pilot carrier along with the message signal so that simple reception is feasible [1], [2]. The pilot carrier may be intentionally produced or it may be inherent in the modulated signals such as the residual carrier in a coherent subcarrier multiplexed system [3]. However, in such a system the performance could be degraded if the states of polarization of the pilot carrier and the message signal are not the same.

A single-mode fiber is actually a two-mode fiber because it can support two orthogonally polarized eigenmodes. Due to imperfections in fiber manufacture and deployment, the propagation constants of the two eigenmodes are in general different and mode coupling occurs as light propagates through a fiber. The different propagation speeds result in polarization mode dispersion, and maximum dispersion happens when the two modes are equally excited. Consequently, an incident linearly polarized wave may become elliptically polarized due to the phase difference between the two eigenmodes. Since the polarization mode dispersion is frequency dependent, the states of polarization of the carrier and the signal in a coherent system with pilot carrier could be different, degrading system performance.

\section{ANALYSIS}

A phenomenological approach conducted in [4] revealed the existence of principal states of polarization (PSP's). It is shown that there exist two orthogonal input PSP's whose corresponding output states of polarization are invariant with frequency to first-order only if there is negligible polarization-dependent loss.

Manuscript received October 26, 1990; revised January 15, 1991. This work was supported in part by the National Science Council of R.O.C., the United Fiber Optic Communication Inc., AT\&T Taiwan Telecommunications Co. Limited, and Taiwan Telecommunication Co. Limited under Contract NSC80-0512-L002-01.

M.-S. Kao is with the Department of Communication Engineering, National Chiao Tung University, Hsinchu, Taiwan, Republic of China.

J. Wu is with the Department of Electrical Engineering, National Taiwan University, Taipei, Taiwan, Republic of China

IEEE Log Number 9143137.
The model provides a simple way to describe polarization mode dispersion without considering the details of fiber birefringence and mode coupling which is useful in practical applications. Here this model is adopted to evaluate the effect of polarization mode dispersion on a coherent system with pilot carrier.

Let $\vec{E}_{s}$ and $\vec{E}_{c}$ denote the signal and the pilot carrier which are linearly polarized along the same direction and are incident on a single-mode fiber. Here we consider the worst case that the two PSP's are equally excited. Let the two PSP's be specified by two unit vectors $\hat{a}_{+}$and $\hat{a}_{-}$, respectively, then $\vec{E}_{s}$ and $\vec{E}_{c}$ can be expressed as

$$
\begin{aligned}
& \vec{E}_{s}(t)=A_{s} e^{j\left(w_{s} t+m(t)+\Psi(t)\right)}\left[\hat{a}_{+}\left(w_{s}\right)+\hat{a}_{-}\left(w_{s}\right)\right] \\
& \vec{E}_{c}(t)=A_{c} e^{j\left(w_{c} t+\Psi(t)\right)}\left[\hat{a}_{+}\left(w_{c}\right)+\hat{a}_{-}\left(w_{c}\right)\right]
\end{aligned}
$$

where $A_{s, c}$ and $w_{s, c}$ denote the coefficients along the two vectors and angular frequencies of the two waves, respectively, and $\vec{E}_{s}(t)$ carries phase information $m(t)$. We assume that the pilot carrier is derived from the signal so that they have the same phase noise $\Psi(t)$. With negligible polarization-dependent loss, the received signal and pilot carrier can be formulated as [4]

$$
\begin{aligned}
\vec{E}_{s}(t)= & A_{s} e^{-\alpha L_{c}} e^{j\left(w_{s} t+m(t)+\Psi(t)\right)}\left[e^{j \phi_{+}\left(w_{s}\right)} \hat{b}_{+}\left(w_{s}\right)\right. \\
& \left.+e^{j \phi_{-}\left(w_{s}\right)} \hat{b}_{-}\left(w_{s}\right)\right] \\
\vec{E}_{c}(t)= & A_{c} e^{-\alpha L} e^{j\left(w_{c} t+\Psi(t)\right)}\left[e^{j \phi_{+}\left(w_{c}\right)} \hat{b}_{+}\left(w_{c}\right)\right. \\
& \left.+e^{j \phi_{-}\left(w_{c}\right)} \hat{b}_{-}\left(w_{c}\right)\right]
\end{aligned}
$$

where $\alpha$ is the fiber loss and $L$ is the length, $\hat{b}_{ \pm}\left(w_{s, c}\right)$ denote the corresponding output states of polarization of $\hat{a}_{ \pm}\left(w_{s, c}\right)$ whereas $\phi_{ \pm}\left(w_{s, c}\right)$ are the corresponding phases. Since the output PSP's are independent of frequency to first-order and the frequency deviation between the two waves under concern is small, therefore in the following we take $\hat{b}_{ \pm}\left(w_{s}\right)=\hat{b}_{ \pm}\left(w_{c}\right)$. The output PSP's are functions of the details of fiber birefringence and the perturbations through the propagation path which are difficult to explicitly express. However, they do preserve the unitary and orthogonal properties [4], written as

$$
\begin{gathered}
\left|\hat{b}_{ \pm}\right|^{2}=1 \\
\hat{b}_{+} \cdot \hat{b}_{-}^{*}=0 .
\end{gathered}
$$

At the receiver, the combined wave $\vec{E}(t)=\vec{E}_{s}(t)+\vec{E}_{c}(t)$ is directly incident on a photodetector (PD) to produce a photocurrent as

$$
\begin{aligned}
i(t) & =\frac{R}{2}|\vec{E}(t)|^{2} \\
& =\frac{R}{2}\left(\left|\vec{E}_{s}\right|^{2}+\left|\vec{E}_{c}\right|^{2}+\vec{E}_{s} \cdot \vec{E}_{c}^{*}+\vec{E}_{c} \cdot \vec{E}_{s}^{*}\right)
\end{aligned}
$$




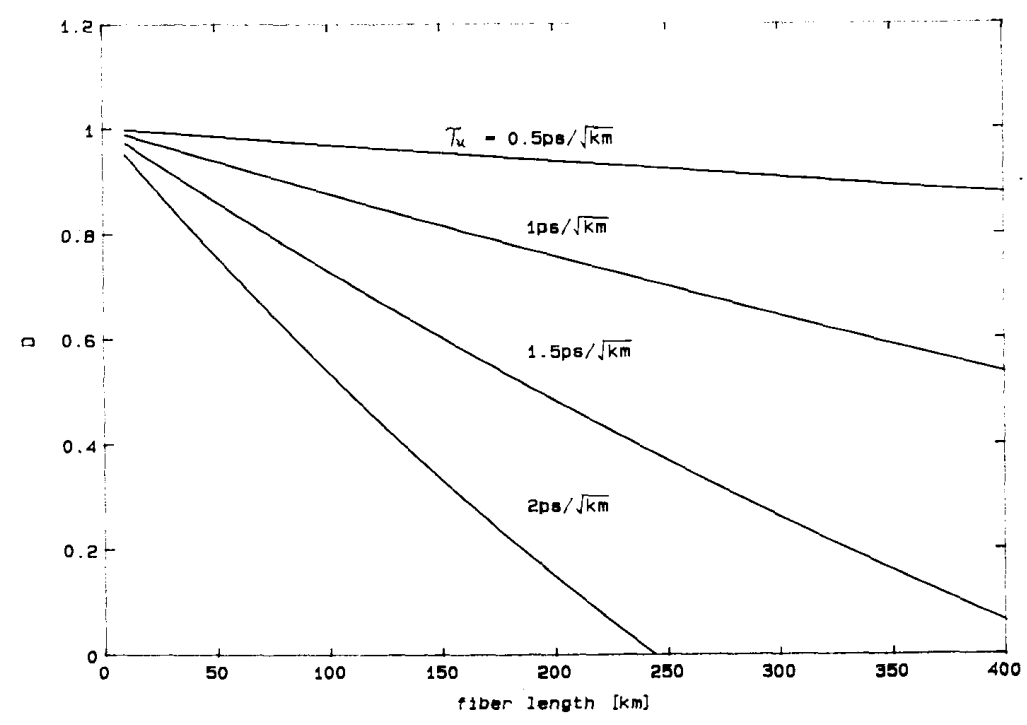

Fig. 1. The degradation factor versus fiber length for several unit differential group delays. $w_{\mathrm{IF}}=2 \pi \times 8 \mathrm{GHz}$

where $R$ is the PD responsivity and the asterisk denotes the complex conjugate. Using the unitary and orthogonal properties of $\hat{b}_{ \pm}$and neglecting the dc terms, the intermediate frequency (IF) current is obtained as

$$
\begin{aligned}
i(t)=R A_{s} A_{c} e^{-2 \alpha L}[ & \cos \left(w_{\mathrm{IF}} t+m(t)+\theta\right) \\
& \left.+\cos \left(w_{\mathrm{IF}} t+m(t)+\theta+\Delta \phi\right)\right]
\end{aligned}
$$

where for simplicity we take $\theta=\phi_{+}\left(w_{s}\right)-\phi_{+}\left(w_{c}\right)$. Also $w_{\mathrm{IF}}$ $=w_{s}-w_{c}$ is the intermediate frequency and $\Delta \phi=\phi\left(w_{s}\right)-$ $\phi\left(w_{c}\right)$ where $\phi\left(w_{s, c}\right)=\phi_{-}\left(w_{s, c}\right)-\phi_{+}\left(w_{s, c}\right)$ are the phase differences between the two PSP's at the two frequencies. We see that the phase noise $\Psi(t)$ is eliminated at the IF signal.

To evaluate the degradation due to polarization mode dispersion, we consider the ideal case when there is no polarization mode dispersion such that the two linearly polarized waves will sustain linear polarization throughout the transmission path. Under such a condition the waves at the receiving end can be written as

$$
\begin{aligned}
\vec{E}_{s}(t)= & A_{s} e^{-\alpha L} e^{j\left(w_{s} t+m(t)+\Psi(t)\right)}\left[e^{j \psi\left(w_{s}\right)} \hat{a}_{+}\left(w_{s}\right)\right. \\
& \left.+e^{j \psi\left(w_{s}\right)} \hat{a}_{-}\left(w_{s}\right)\right] \\
\vec{E}_{c}(t)= & A_{c} e^{-\alpha L} e^{j\left(w_{c} t+\Psi(t)\right)}\left[e^{j \psi\left(w_{c}\right)} \hat{a}_{+}\left(w_{c}\right)\right. \\
& \left.+e^{j \psi\left(w_{c}\right)} \hat{a}_{-}\left(w_{c}\right)\right]
\end{aligned}
$$

where we assume the two PSP's are preserved through the fiber and the two PSP's have the same phases. Note that the phases of the PSP's at the two frequencies $\psi\left(w_{s}\right)$ and $\psi\left(w_{c}\right)$ are in genera frequency dependent. The combined wave $\vec{E}(t)=\vec{E}_{s}(t)+\vec{E}_{c}(t)$ is similarly incident on a PD. Again $\hat{a}_{+}$preserve the unitary and orthogonal properties so that the resultant IF current can be expressed as

$$
i(t)=2 R A_{s} A_{c} e^{-2 \alpha L} \cos \left(w_{\mathrm{IF}} t+m(t)+\theta^{\prime}\right)
$$

where $\theta^{\prime}=\psi\left(w_{s}\right)-\psi\left(w_{c}\right)$.
It is evident by observing (8) and (11) that the constant angles $\theta^{\prime}$ and $\theta$ are irrelevant to the IF signal but $\Delta \phi$ is obviously critical. For $\Delta \phi=0$ we see that the IF signal is the same as that of the ideal case and it deviates from the ideal case if $\Delta \phi \neq 0$. We can reformulate (8) as

$$
\begin{array}{r}
i(t)=2 R A_{s} A_{c} e^{-2 \alpha L} \cos \left(w_{\mathrm{IF}} t+m(t)+\theta+\frac{\Delta \phi}{2}\right) \\
\cdot \cos \left(\frac{\Delta \phi}{2}\right)
\end{array}
$$

which shows that the IF signal degrades by a factor $\cos \frac{\Delta \phi}{2}$ compared to the ideal case. $\Delta \phi$ can be estimated from the differential group delay of the two PSP's, denoted by $\tau_{p}$, as [5]

$$
\tau_{p}=\frac{d \phi}{d w}
$$

where $\phi=\phi_{-}(w)-\phi_{+}(w)$ is the phase difference between the two PSP's. From (13) we have

$$
\Delta \phi=\phi\left(w_{s}\right)-\phi\left(w_{c}\right)=\int_{w_{c}}^{w_{s}} \tau_{p} d w .
$$

From the measured results [5] $\tau_{p}$ over a small frequency range (few gigahertz) is essentially constant, therefore $\Delta \phi$ is approximately given as

$$
\Delta \phi \simeq \tau_{p} w_{1 \mathrm{~F}} .
$$

A statistical treatment revealed that $\tau_{p}$ is proportional to the square root of the fiber length if the length is far larger than the correlation length of perturbations [6]. Let $\tau_{u}$ denote the differential group delay for unit square-root fiber length. We can define a degradation factor due to polarization mode dispersion 


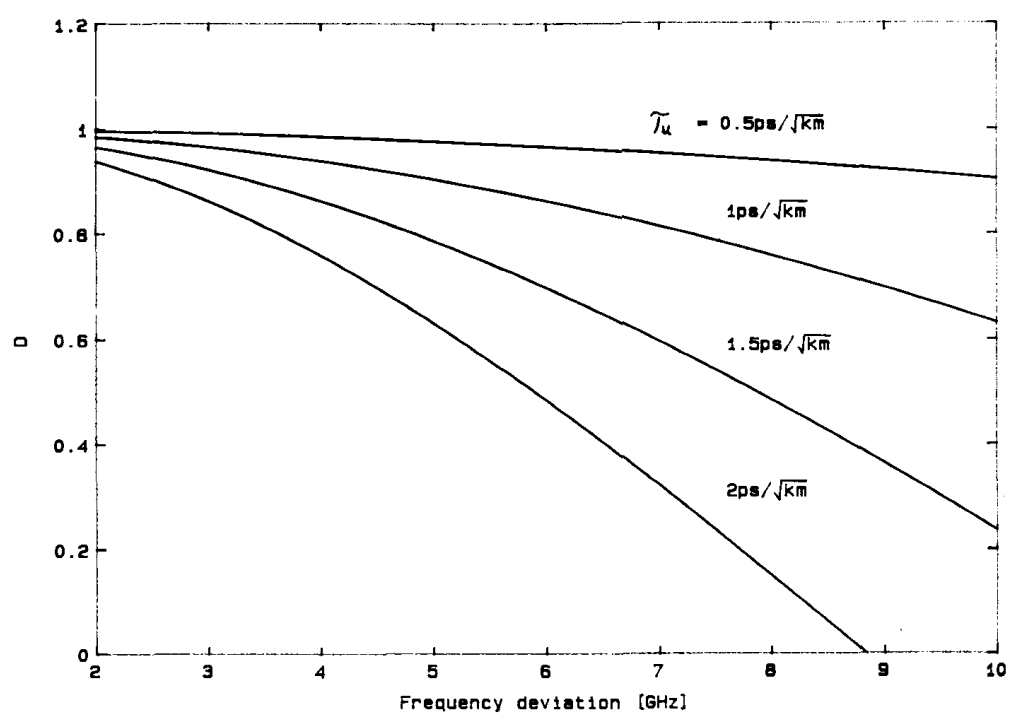

Fig. 2. The degradation factor versus frequency deviation between the signal and the pilot carrier. $L=200 \mathrm{~km}$.

as

$$
D=\cos \left(\frac{\tau_{u} w_{\mathrm{IF}} \sqrt{L}}{2}\right)
$$

where $L$ is the fiber length. Hence the performance degradation can be easily estimated.

\section{Discussion AND Conclusion}

Fig. 1 shows the relation between $D$ and fiber length for several $\tau_{u}^{\prime} s$ with $w_{\mathrm{IF}}=2 \pi \times 8 \mathrm{GHz}$. We see that for $\tau_{u}=0.5$ $\mathrm{ps} / \sqrt{\mathrm{km}}$, there is little degradation even for $400 \mathrm{~km}$ fiber length. However, serious degradation occurs if $\tau_{u}$ becomes large. For $\tau_{u}=2 \mathrm{ps} / \sqrt{\mathrm{km}}, D$ decreases rapidly as fiber length increases. For example $D$ decreases to 0.5 for $120 \mathrm{~km}$ fiber length and it is zero for $245 \mathrm{~km}$ fiber length. Thus, the polarization mode dispersion may limit the system transmission distance if large $\tau_{u}$ exists. We further plot $D$ as a function of the frequency deviation between the signal and the pilot carrier in Fig. 2 for $L=200 \mathrm{~km}$. Again it is seen that little degradation occurs for $\tau_{u}=0.5 \mathrm{ps} / \sqrt{\mathrm{km}}$ within a $10 \mathrm{GHz}$ frequency deviation but the degradation becomes significant for large $\tau_{u}$.

From above results we conclude that the polarization mode dispersion indeed degrades the IF signal of a coherent optical system using pilot carrier. If the differential group delay between the two PSP's is small there is little performance degradation even for long transmission distance and large frequency deviation between the signal and the pilot carrier. On the other hand, the IF signal may be seriously degraded if the differential group delay of the two PSP's is large. In this case both the system transmission distance and the frequency deviation between the signal and the pilot carrier may be limited. The differential group delay between the two PSP's is a consequence of nonideal fiber geometry and perturbations along the transmission path. Thus, if there exists large differential group delay between the two PSP's, either because of fiber geometry or because of perturbations along the transmission fiber, the use of pilot carrier may not be advantageous since the IF signal could be seriously degraded.

In conclusion we have evaluated the performance degradation due to polarization mode dispersion in a coherent optical system with pilot carrier. Using the model of principal states of polarization, the IF signal is obtained and compared to the ideal case without polarization mode dispersion. A simple expression is derived which can be used to evaluate the performance degradation. The results show that the IF signal can be severely degraded if large differential group delay exists between the two principal states of polarization. Hence the use of pilot carrier may not be advantageous for a system with large differential group delay between the two principal states of polarization.

\section{REFERENCES}

[1] J. A. Arnaud, "Enhancement of optical receiver sensitivity by amplification of the carrier," IEEE J. Quantum Electron., vol. QE-4, pp. 893-899, Nov. 1968.

[2] C. G. Atkins, D. Cotter, D. W. Smith, and R. Wyatt, "Application of Brillouin amplification in coherent optical transmission," IEE Electron. Lett., vol. 22, no. 10, pp. 556-558, May 1986.

[3] R. Gross, R. Olshansky, and P. Hill, " 20 channel coherent FSK system using subcarrier multiplexing," IEEE Photon. Technol. Lett., vol. 1, pp. 224-226, Aug. 1989.

[4] C. D. Poole and R. E. Wagner, "Phenomenological approach to polarization dispersion in long single-mode fibers," IEE Electron. Lett., vol. 22, no. 19, pp. 1029-1030, Sept. 1986.

[5] C. D. Poole, N. S. Bergano, R. E. Wagner, and H. J. Schulte, "Polarization dispersion and principal states in a $147-\mathrm{km}$ undersea lightwave cable," J. Lightwave Technol., vol. 6, pp. 1185-1190, July 1988 .

[6] F. Curti, B. Daino, G. D. Marchis, and F. Matera, "Statistical treatment of the evolution of the principal states of polarization in single-mode fibers," J. Lightwave Technol., vol. 8, pp. 1162-1166, Aug. 1990. 